\title{
Autoestima e desempenho escolar em matemática: contribuições teóricas sobre a problematização das relações entre cognição e afetividade
}

\section{Self-esteem and school achievement in mathematics: theoretical contributions to the framing of the relationship between cognition and affection}

\author{
Izabel Hazin ${ }^{1}$ \\ Cristina Frade ${ }^{2}$ \\ Jorge Tarcísio da Rocha Falcão ${ }^{3}$
}

\begin{abstract}
RESUMO
Este estudo investigou conexões existentes entre aspectos afetivos e cognitivos no contexto da aprendizagem escolar, notadamente em termos das relações entre autoestima e desempenho em matemática. Participaram do estudo vinte alunos do Ensino Fundamental II (5a. Série) de uma escola pública da cidade de Recife PE. O estudo foi realizado em duas etapas: 1. Aplicação do teste HTP aos alunos participantes, com o objetivo de identificar o nível de autoestima dos mesmos. Ao final desta etapa foram
\end{abstract}

1 Doutora em Psicologia Cognitiva pela Universidade Federal de Pernambuco, linha de pesquisa neuropsicologia do desenvolvimento e da aprendizagem. Departamento de Psicologia Centro de Ciências Humanas, Letras e Artes (CCHLA) - Universidade Federal do Rio Grande do Norte (UFRN). E-mail: izabel.hazin@gmail.com.

2 Doutora em educação pela UFMG, linha de pesquisa em educação matemática. Centro Pedagógico da Escola de Educação Básica e Profissional da Universidade Federal de Minas Gerais (UFMG).

3 Doutor em psicologia da aprendizagem e do desenvolvimento pela Université de Paris 5/ René Descartes/ Sorbonne, linha de pesquisa em competências cognitivas complexas na escola e no trabalho. Departamento de psicologia - Centro de Ciências Humanas, Letras e Artes (CCHLA) - Universidade Federal do Rio Grande do Norte (UFRN). 
constituídas duplas homogêneas e heterogêneas contemplando as variáveis gênero e nível de autoestima. 2) Resolução em duplas de instrumento de avaliação matemática. Os dados foram analisados partindo-se de técnicas categoriais e multidimensionais. Observou-se que o nível alto de autoestima relaciona-se a padrões de interação forte entre os componentes da dupla e ao desempenho matemático sem dificuldades. O nível baixo de autoestima vincula-se a padrões de interação fraco e ao desempenho matemático caracterizado por dificuldades. Tais dados são discutidos no contexto da teorização acerca das relações entre cognição e afetividade, bem como em termos de seus desdobramentos para a prática escolar de ensino da matemática.

Palavras-chave: Autoestima; desempenho escolar em matemática; cognição; afetividade.

\begin{abstract}
The research reported here aimed to analyze the relations between affect and cognition in school learning, focusing on mathematical learning at school. Twenty-seven elementary level (5th year) students from a public school at Recife (PE) took part in this research, which covered two stages: 1. Application of HTP Test to the participant students, aiming to identify their self-esteem levels. This procedure allowed the constitution of homogenous and heterogeneous dyads according to gender and self-esteem level. 2. Application to the dyads of an instrument of evaluation of mathematics performance, to be solved by the students cooperatively. Data collected during these two stages were analyzed with the help of multidimensional descriptive statistical tools, which allowed the interpretation of a link between self-esteem and mathematical performance: low and high levels of self-esteem were respectively associated to low and high levels of performance in school mathematics. These data are discussed in terms of both theoretical and pedagogical consequences for mathematics learning.

Key-words: self-esteem; school performance in mathematics; cognition; affect.
\end{abstract}

\title{
Introdução
}

O presente estudo investigou as relações existentes entre o nível de autoestima e o desempenho escolar em matemática de crianças do $6^{\circ}$. ano de uma 
escola pública da cidade de Recife-PE. Partiu-se aqui do pressuposto segundo o qual a abordagem integradora de aspectos cognitivos e afetivos, no contexto da psicologia em geral e da psicologia da educação matemática em particular, é possível e altamente desejável. Para fins operacionais, escolheu-se a construção conceitual em matemática como representante da cognição e a autoestima das crianças envolvidas no processo, como representante da afetividade (para uma discussão acerca da pertinência destas escolhas, ver NEVES; CARVALHO, 2006; IGNACIO; NIETO; BARONA, 2006; HANULLA; EVANS; PHILIPPOU; ZAN, 2004; ZAN; BROWN; EVANS; HANNULA, 2006; MALMIVUORI, 2004; HAZIN; DA ROCHA FALCÃO, 2001).

Tal pressuposto pode ser justificado em termos de três argumentos centrais: a) Tendo em vista que os afetos envolvem em determinada medida consciência e conhecimento, trata-se de processo cognitivo; inversamente, tendo em vista que muitos conteúdos da cognição têm uma direção motivacional e um colorido emocional, trata-se de processo afetivo; b) O psiquismo humano não pode ser limitado à esfera da experiência consciente: o sistema cognição-afetividade é estruturado por componentes advindos de ambos os domínios e só pode ser acessado mediante a mobilização desses componentes de forma integrada. Entretanto, o acesso nunca se dá de forma completa, pois algumas partes do sistema são inconscientes (SCHLÖGLMANN, 2004); c) Conforme proposto por A. Damásio, a tradicional separação topológico-neurológica entre uma região neocortical dedicada à racionalidade e um substrato subcortical dedicado ao controle automático, neurovegetativo e hormonal parece superada (DAMÁSIO, 2003; 1996). Tal discussão é complementada pelas considerações de G. Lakoff e R. Núñez acerca da corporeidade (embodiement) da mente (LAKOFF; NÚÑEZ, 2000). Esses autores chamam a atenção para o fato de que corpos, cérebros e experiência cotidiana no mundo estruturam a conceptualização e racionalidade humanas. No que diz respeito à matemática, Lakoff e Núñez observam ainda que esta deveria ser considerada como um produto complexo das capacidades neurais de nossos cérebros, da natureza de nossos corpos, de nossa evolução, de nosso meio ambiente e de nossa longa história sociocultural.

A construção de relações entre cognição e afetividade é uma tarefa teórica apenas iniciada em psicologia. A tradição, desde Descartes, de cisão entre aspectos cognitivos (res cogitans) e somático-afetivos (res extensa) propiciou o contexto filosófico e psicológico de onde surgiram sistemas teóricos com ênfases em um ou outro desses domínios.

No que diz respeito especificamente ao estudo psicológico da cognição, foi necessário, num primeiro momento, romper o interdito behaviorista da consideração de construtos inferidos (p. ex. esquemas mentais), conducentes a um mentalismo visto como fatal à abordagem objetiva e científica do objeto 
psicológico (índices comportamentais diretamente acessáveis). Tal movimento de instauração de um espaço para a consideração da cognição é historicamente devedor da psicologia da Gestalt, notadamente das reflexões de Max Wertheimer, em que se faz uma distinção crucial entre pensamento produtivo e reprodutivo, sendo somente este último explicável pelos padrões de modificação de vínculos estímulo-resposta (WERTHEIMER, 1959).

Piaget, nessa trilha, propõe uma epistemologia genética em que, a partir da adaptação, um processo central de natureza biológica, o conhecimento humano evoluiria ao longo de estágios rumo a formas de funcionamento cada vez mais funcionais e adaptativas, posto que flexíveis, descentradas e reversíveis (PIAGET, 1973; 1990). Adicionalmente, dois aspectos merecem especial destaque na proposição teórica piagetiana: em primeiro lugar, todo organismo possui uma estrutura permanente que, por sua vez, é modificada quando posta sob a influência do meio, mas jamais destruída; nesse sentido, todo conhecimento diz respeito à assimilação de um dado exterior a estruturas do indivíduo. Segundo, os fatores normativos do pensamento correspondem biologicamente a uma necessidade de equilíbrio por autorregulação. Nessa perspectiva, ser inteligente é estabelecer com o meio uma interação equilibrada e autorregulada, em que a afetividade é admitida como inextricavelmente imbricada:

(...) a afetividade constitui a energética das condutas, cujo aspecto cognitivo se refere apenas às estruturas. Não existe, portanto, nenhuma conduta, por mais intelectual que seja, que não comporte, na qualidade de móveis, fatores afetivos; mas, reciprocamente, não poderia haver estados afetivos sem a intervenção de percepções ou compreensão, que constituem a sua estrutura cognitiva. A conduta é, portanto, una, mesmo que, reciprocamente, não tome aquelas em consideração: os dois aspectos afetivo e cognitivo são, ao mesmo tempo, inseparáveis e irredutíveis. (PIAGET, 1980, p.135).

Ainda que se observe tal papel de “combustível” das "estruturas” de funcionamento cognitivo (DOLLE, 1979; 1993), a afetividade não aparece como variável explicativa relevante em nenhum dos trabalhos oriundos da tradição piagetiana de abordagem da cognição (SCHLÖGLMANN, 2007). Tal constatação, sugere Souza (2003), deve-se à prioridade de Piaget pela busca e descrição das estruturas ou formas de organização da inteligência.

Outra contribuição importante da psicologia acerca da discussão sobre a inseparabilidade entre cognição e afeto advém de Vygotsky (1993). Para ele, a 
afetividade parece não ser meramente uma questão de "combustível” das estruturas do funcionamento cognitivo; ela não só participaria do funcionamento mental, como também estaria integrada/acoplada a ele:

Quem separa desde o começo o pensamento do afeto fecha para sempre a possibilidade de explicar as causas do pensamento, porque uma análise determinista pressupõe descobrir seus motivos, as necessidades e interesses, os impulsos e tendências que regem o movimento do pensamento em um ou outro sentido. De igual modo, quem separa o pensamento do afeto, nega de antemão a possibilidade de estudar a influência inversa do pensamento no plano afetivo, volitivo da vida psíquica, porque uma análise determinista dessa última inclui tanto atribuir ao pensamento um poder mágico capaz de fazer depender o comportamento humano única e exclusivamente de um sistema interno de indivíduos, como transformar o pensamento em um apêndice inútil do comportamento, em uma sombra sua desnecessária e impotente. (VYGOSTKY, 1993, p. 25).

Esse mesmo autor argumenta ainda que nossos sentimentos (afetos) também fazem parte do processo de construção cultural da significação, por meio do qual aprendemos e apreendemos dialeticamente, do mundo que nos cerca (cultura), as formas de ver e agir sobre esse mundo. Sendo assim, a afetividade é um componente do sistema conceitual que nos foi dado e imposto pelo meio que nos rodeia (VYGOTSKY, 2001).

Nessa mesma direção de exploração da cognição em contato com aspectos afetivos e motivacionais, cabe ressaltar a contribuição fundamental da psicanálise freudiana (FREUD, 1970-1977). Freud ressalta o aspecto energético da conduta como central na sua teoria. A pulsão, introduzida na teoria freudiana em $1915^{4}$, é tomada como conceito limítrofe entre a vida mental e o corpo biológico, sendo tanto a representante mental dos estímulos provenientes do organismo, como uma medida das exigências feitas à mente em consequência de sua conexão com o corpo. Para a psicanálise, a meta última de qualquer pulsão seria a descarga de energia, a redução de tensão, que pode ser alcançada por vias transversas, como é o caso do deslocamento da energia de um objeto para outro, quando o objeto original do desejo é inacessível. Ora, se anteriormente se fez alusão à dificuldade de visualização, em Piaget, de um papel razoavelmente delimitado da afetividade enquanto variável ou conjunto de variáveis partícipes no que diz

4 Vide notadamente a obra As pulsões e suas vicissitudes (FREUD, 1970-1977). 
respeito à produção de significado inerente à intelecção humana, em Freud a motivação para tal intelecção é assimilada à esfera pulsional, do que resulta seu enquadramento enquanto impulso epistemofílico sublimatório.

Freud, Piaget e Vygotsky não são aqui citados como referências que esgotam o campo de reflexões acerca dos eventuais contatos entre cognição e afetividade, mas podem ser considerados, cada um em seu contexto de produção, como exemplos do quão difícil é ultrapassar a cisão a que aludíamos no início da presente seção introdutória. Como consequência de tais dificuldades, os esforços de pesquisa concreta em psicologia, voltadas para a produção de dados que ensejem a consideração de afetividade e cognição enquanto aspectos efetivamente contemplados de forma integrada são escassos (ARAÚJO et al., 2003). O presente trabalho representa um esforço empírico nessa direção, conforme detalhamento explicitado nas seções seguintes.

\section{Metodologia}

Amostra

Trabalhamos nesta pesquisa com crianças na faixa etária de 12 a 14 anos, alunos da $5^{\text {a }}$ série do Ensino Fundamental II de uma escola pública estadual, na cidade do Recife. A opção pela $5^{\text {a }}$ série decorreu de especificidade desse nível escolar, segundo dados de 2001 do Sistema Nacional de Avaliação da Educação Básica (Saeb) (MEC, 2002), que apontam tal momento da escolarização como sendo caracterizado por uma queda média significativa no desempenho e consequente taxa de aprovação em matemática. Paralelamente a esses dados, A. Fierro pontua que vários estudos mencionam uma queda da autoestima por volta dos 12-13 anos (FIERRO, 1996), o que dá interesse adicional à opção pela $5^{\mathrm{a}}$ série, já que esta corresponde ao nível escolar esperado para tal faixa etária.

\section{Procedimento}

A pesquisa compreendeu três etapas principais. A etapa 1 consistiu na aplicação individual da técnica projetiva $\mathrm{HTP}^{5}$, que consiste da elaboração de desenhos com os temas Casa, Árvore e Pessoa, seguidos de inquérito. Os

5 O referido teste foi utilizado de acordo com os critérios de administração e interpretação descritos por E. Hammer (HAMMER, 1991). 
desenhos são realizados em duas fases, a saber, a fase acromática, onde a criança utiliza lápis HB preto, e a fase cromática onde lápis coloridos são disponibilizados para que a criança efetue os desenhos. Essa etapa visou avaliar o nível de autoestima dos sujeitos inicialmente disponíveis para participação. O instrumento foi aplicado a todos os alunos das duas turmas de $5^{\text {a }}$ série do turno da manhã da escola escolhida. A partir desse procedimento de testagem, o qual envolveu uma amostra inicial de 81 crianças, chegou-se a um grupo final de 20 sujeitos, sendo dez meninos e dez meninas. Esses 20 sujeitos foram agrupados em duplas homogêneas e heterogêneas, em função do nível de autoestima e gênero ${ }^{6}$, conforme ilustrado pelo quadro 1.

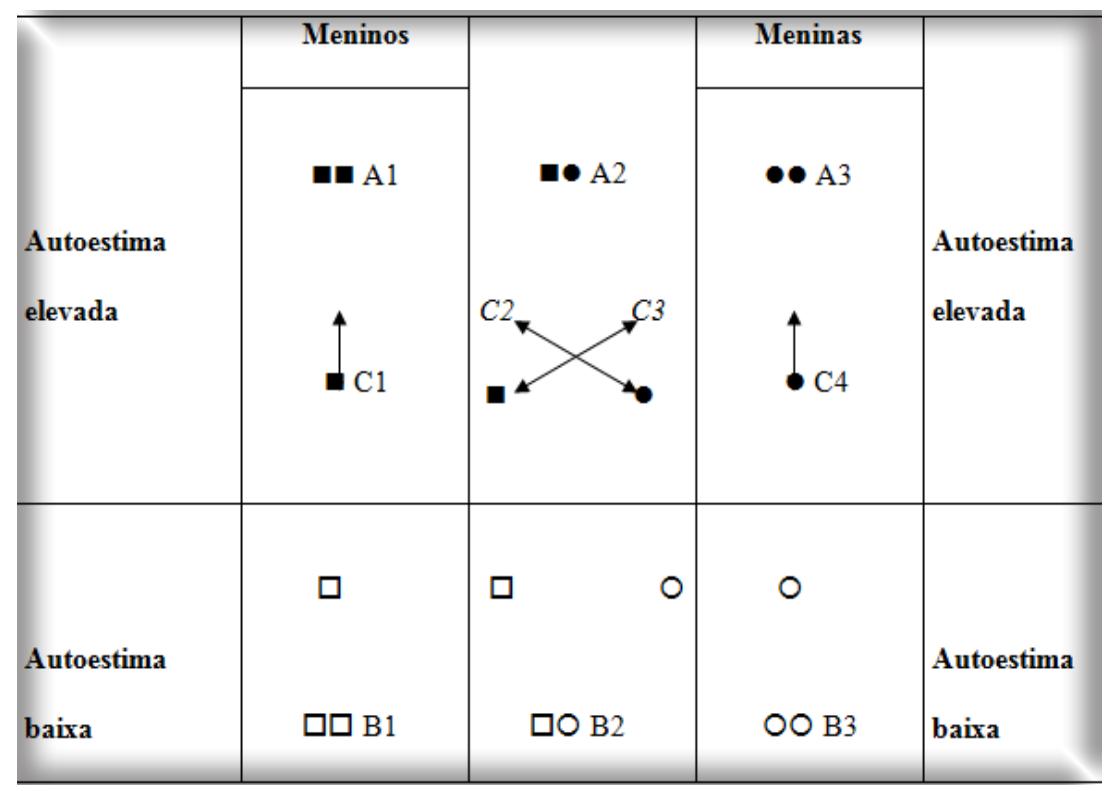

Quadro 1: Plano de constituição das duplas de sujeitos em função do gênero e do nível de autoestima detectado.

A etapa 2 consistiu da aplicação de questionário de avaliação desenvolvido inicialmente pela equipe da Secretaria de Educação da Cidade do Recife e

6 A consideração da variável gênero na constituição das duplas partiu do interesse em se verificar empiricamente uma eventual diferenciação de desenvolvimento biológico e psicoafetivo entre meninos e meninas na fase da adolescência, com eventuais repercussões em termos de desempenho matemático escolar. 
implementado pelo Núcleo de Pesquisa em Avaliação de Pernambuco (Nape) UFPE (DA ROCHA FALCÃO, 1997). Tal questionário foi desenvolvido visando uma avaliação global de desempenho em matemática ao final do primeiro ciclo do ensino fundamental (final da $4^{\mathrm{a}}$ série), conforme perfil reproduzido nquadro 2 abaixo. A resolução do questionário, pelas duplas, aconteceu em uma única sessão de trabalho.

\begin{tabular}{|c|c|}
\hline $\begin{array}{l}\text { Aspecto matemático visado } \\
\text { [Gn =Grupo] e número de questões } \\
\text { no teste }\end{array}$ & Exemplo de um item do teste \\
\hline $\begin{array}{l}\mathbf{G}_{1}=\text { Problemas de estrutura aditiva } \\
-3 \text { questões }\end{array}$ & $\begin{array}{l}\text { Um avião deve percorrer uma distância } \\
\text { de } 962 \mathrm{~km} \text { em duas etapas: na primeira etapa ele } \\
\text { percorrerá uma distância de } 642 \mathrm{~km} \text {. Quantos } \\
\text { quilômetros este avião terá de percorrer na } \\
\text { segunda etapa? }\end{array}$ \\
\hline $\begin{array}{l}\qquad \mathbf{G}_{2}=\text { Problemas de estrutura multi- } \\
\text { plicativa } \\
-3 \text { questões }\end{array}$ & $\begin{array}{l}\text { Uma canoa pode transportar um máximo } \\
\text { de } 200 \mathrm{~kg} \text { por viagem. Qual seria o número mí- } \\
\text { nimo de viagens que esta canoa deveria fazer de } \\
\text { forma a transportar } 8 \text { pessoas, cada uma delas } \\
\text { pesando } 60 \mathrm{~kg} \text { ? }\end{array}$ \\
\hline $\begin{array}{l}\mathbf{G}_{3}=\text { Questões envolvendo o uso de } \\
\text { algoritmos } \\
-2 \text { questões }\end{array}$ & $\begin{array}{r}\text { Faça estas contas: } \quad 3529 \div 15 \\
3847+5+98\end{array}$ \\
\hline $\begin{array}{l}\qquad \mathrm{G}_{4}=\text { Questões envolvendo a com- } \\
\text { preensão do sistema decimal de contagem } \\
\text { e notação } \\
\text { - } 2 \text { questões }\end{array}$ & $\begin{array}{l}\text { O número oitocentos e dois escrito em } \\
\text { algarismos hindu-arábicos é: }\end{array}$ \\
\hline $\begin{array}{l}\mathbf{G}_{5}=\text { Questões de geometria } \\
-3 \text { questões }\end{array}$ & $\begin{array}{l}\text { Olhe atentamente para esta peça de jogo de } \\
\text { encaixe: como seria vista esta peça se olhada de } \\
\text { cima para baixo? [Opções mostradas abaixo] }\end{array}$ \\
\hline
\end{tabular}

(Continua) 
(Conclusão)

\begin{tabular}{|c|c|}
\hline $\begin{array}{l}\text { G6= Questões envolvendo frações } \\
\text { - } 3 \text { questões }\end{array}$ & $\begin{array}{l}\text { A figura abaixo representa uma barra de } \\
\text { chocolate. Preencha de preto a parte correspon- } \\
\text { dente, nesta figura, à adição seguinte: } 2 / 6+1 / 6 \\
\text { da barra de chocolate. } \square \square \square \square\end{array}$ \\
\hline $\begin{array}{l}\text { G7= Questões referentes à compreensão } \\
\text { de gráficos estatísticos descritivos e medidas } \\
\text { usuais. } \\
\text { - } 4 \text { questões } \\
\text { No gráfico à direita, quantos sorvetes } \\
\text { foram vendidos em outubro, considerando que } \\
\text { esta quantidade foi o dobro de setembro? }\end{array}$ & $\begin{array}{l}\text { Quant. de } \\
\text { sorvetes }\end{array}$ \\
\hline
\end{tabular}

Quadro 2: Perfil básico de questões propostas no questionário NAPE

Finalmente, a etapa 3 compreendeu a categorização e análise dos protocolos gerados pelas duplas, em termos de dois itens básicos: o desempenho no instrumento de avaliação em matemática escolar (escore de acertos) e o tipo de procedimento mobilizado para a resposta à questão. Realizada tal categorização, os dados foram, então, analisados em ambiente de análise descritiva multidimensional, complementada por análise clínica.

A seção seguinte discute os resultados obtidos em função do planejamento metodológico acima resumido.

\section{Resultados}

O presente estudo teve como objetivo central buscar conexões entre aspectos cognitivos (desempenho em matemática) e afetivos (autoestima) no âmbito de determinado contexto social de produção de significado (matemática escolar). A análise dos dados obtidos aqui apresentada restringe-se a dois momentos de análise sequenciados e inter-relacionados: (1) Etapa preliminar de análise dos protocolos referentes à técnica projetiva do desenho da Casa-Árvore-Pessoa (HTP), de modo a se formarem duplas cuja constituição levasse em conta a manipulação da variável “autoestima”, conforme explicitado no quadro 2 abaixo; (2) Análise descritiva multidimensional, fundada em procedimento informatizado 
de categorização, a partir de duas grades de classificação: produção das duplas para as questões-problema do questionário NAPE e organização das variáveis descritivas auxiliares.

\section{Etapa preliminar de análise das produções no teste HTP}

A utilização do teste HTP visou selecionar os integrantes das duplas, que iriam, posteriormente, responder ao Questionário NAPE. Tal seleção teve como critério o nível de autoestima apresentado pelos integrantes das duplas. A técnica projetiva do desenho da Casa-Árvore-Pessoa (HTP) fornece subsídios para a avaliação dos aspectos dinâmicos da personalidade, que surgem nos desenhos produzidos. A análise do HTP pode ser feita nos níveis quantitativo e qualitativo, partindo-se dos componentes expressivos e de conteúdo, apresentados nas fases acromática e cromática dos desenhos e nos respectivos inquéritos.

Neste trabalho, optou-se pela análise do HTP exclusivamente a partir do nível qualitativo, já que a discussão acerca do nível de QI apresentado pelas crianças não ocupava um lugar dentro do nosso estudo. Para tal análise foi utilizada a grade básica de critérios de interpretação do HTP proposta por E. Hammer (1991), levando em consideração os aspectos expressivos e de conteúdo, presentes nos desenhos e nos inquéritos, diretamente relacionados ao baixo nível de autoestima (para uma descrição completa de tais critérios, ver HAMMER, 1991; HAZIN, 2001).

\section{Análise descritiva multidimensional}

Os dados referentes à caracterização e atividade de resolução de problemas matemáticos das duplas constituintes da amostra foram analisados a partir de grades de categorização voltadas para dois conjuntos de aspectos: um primeiro conjunto de categorias baseou-se em variáveis descritivoqualificativas das duplas em si, e um segundo conjunto abarcou aspectos auxiliares referentes ao procedimento matemático mobilizado, em termos de obstáculos e estratégias na resolução da cada uma das vinte questões do questionário NAPE.

A análise dos dados obtidos a partir da caracterização e da atividade das duplas na resolução do questionário NAPE, categorizados a partir das grades mencionadas anteriormente, foi realizada utilizando-se algoritmo informatizado de análise descritiva multidimensional, dispondo os dados coletados em uma 
Classificação Ascendente Hierárquica ${ }^{7}$. Tal procedimento de análise permite-nos lançar um olhar sobre os dados da pesquisa sob o prisma de diferentes perspectivas simultaneamente. Diferentemente da análise unidimensional, que nos oferece a possibilidade de estabelecermos conexões apenas entre duas dimensões de cada vez (esquema clássico de estabelecimento de nexo e inferência de relação causal entre variáveis independentes (VIs) e dependentes (VDs)), a análise multidimensional promove a associação de várias dimensões ao mesmo tempo, de forma a caracterizar o fenômeno em estudo de forma mais abrangente e sistêmica (ROAZZI, 1995).

A Classificação Ascendente Hierárquica procede agrupando os sujeitos participantes da amostra (no caso do nosso estudo, as duplas) estruturando classes principais erguidas através da força de partição das categorias referentes às variáveis caracterizadoras de cada sujeito participante. O objetivo maior dessa ferramenta é agrupar indivíduos em determinadas classes o mais possível homogêneas, de acordo com critérios preestabelecidos, a saber, categorias classificatórias nominais (grades de categorização). O instrumento de análise opera, por fim, uma partição de classes dita hierárquica, ou seja, que parte de uma partição mais abrangente ${ }^{8}$ para as mais específicas no interior de cada grande grupo $^{9}$. As subseções abaixo passam a detalhar tal análise.

\section{Dados referentes às variáveis descritivas}

As variáveis descritivas utilizadas nesta primeira análise (ver Figura 1, árvore (1)) foram GÊNERO (masculino e feminino), NÍVEL DE AUTOESTIMA DAS DUPLAS (elevado e baixo), PADRÃO DE LIDERANÇA demonstrado pelos membros da dupla (ausência ou presença de um líder) e PADRÃO DE INTERAÇÃO dos membros da dupla (cooperação, competição ou submissão).

7 Para operacionalização de tal análise, utilizou-se o procedimento informatizado CAHVOR da bateria ADDAD (Association pour le Développement de l'Analyse de Données), precedido pelo programa de preparação dos dados DS-3, desenvolvido por Denys Corroyer. Para referência acerca dessas ferramentas de análise de dados, ver Fénelon (1981 ).

8 Por exemplo, uma partição entre “cães” e "gatos”.

9 No âmbito da partição anterior, uma subpartição dos cães e dos gatos em raças específicas. 


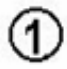

10 㣽个总怰

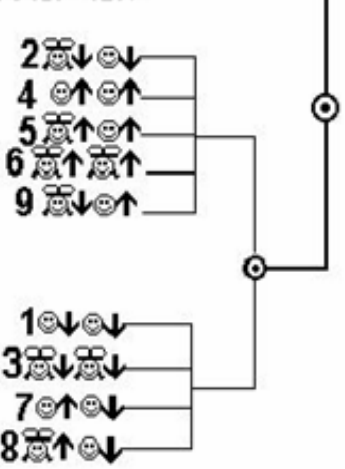

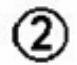

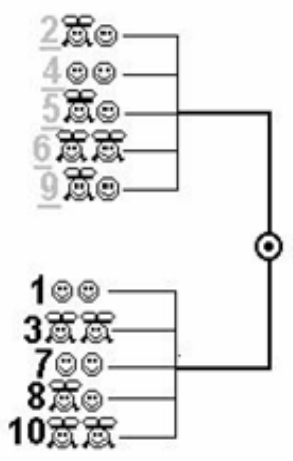

\begin{tabular}{|c|c|c|c|}
\hline $\begin{array}{l}\text { Meninos } \\
\text { Meninas }\end{array}$ & $\begin{array}{l}\downarrow \text { Auto estima baixa } \\
\uparrow \text { Auto estima elevada }\end{array}$ & Teste NAPE & $\begin{array}{l}\text { Baixo nivel de dificuldade } \\
\text { Alto nivel de dificuldade }\end{array}$ \\
\hline
\end{tabular}

Figura 1: Representação da árvore hierárquica (CAH) produzida a partir das variáveis descritivas e de desempenho no teste NAPE utilizadas no estudo.

A representação esquemática obtida, através da classificação hierárquica ascendente, dos principais agrupamentos de duplas, segundo as variáveis descritivo-qualificativas acima referidas, é apresentada na Figura 1 - árvore (1) acima. Percebemos que no primeiro nível de partição, a dupla 10 surge isolada das demais duplas. No segundo nível de estruturação temos dois conjuntos, um formado pelas duplas 2, 4, 5, 6 e 9 e outro formado pelas duplas 1, 3, 7 e 8. A análise clínica cuidadosa dos protocolos dessas duplas permite presumir que a segunda partição realizada no interior da amostra guiou-se basicamente pelas categorias de PADRÃO DE INTERAÇÃO e NÍVEL DE AUTOESTIMA: temos um conjunto de duplas $(2,4,5,6,9)$ caracterizadas basicamente por apresentarem padrão de interação de tipo cooperativo e autoestima elevada; o outro conjunto de duplas (1, 3, 7 e 8) apresenta padrão de interação competitivo/não cooperativo e autoestima baixa. As demais categorias de análise não obtiveram, em nenhuma de suas modalidades, um valor de contribuição acima da média, o que nos leva a crer que são critérios sem força para operarem uma partição entre as duplas. 


\section{Dados referentes ao questionário NAPE}

A representação esquemática obtida através da classificação ascendente hierárquica dos principais agrupamentos de duplas segundo os procedimentos matemáticos por estas adotados, utilizados na categorização dos dados produzidos na resolução do questionário NAPE, é visualizada na figura 1, árvore classificatória (2). Percebemos que no primeiro nível de estruturação temos dois conjuntos, um formado pelas duplas 2, 4, 5, 6 e 9 e outro formado pelas duplas 1, 3, 7, 8 e 10. A análise dos protocolos dessas duplas sugeriu uma forte oposição das modalidades de resposta vinculadas à realização da questão com baixo nível de dificuldades (duplas 4, 5, 6 e 9), em oposição ao outro conjunto de duplas (1, 3, 7, 8 e 10), caracterizado por alto nível de dificuldade (e demanda elevada de auxílio por parte do experimentador) na resolução das questões propostas. Ao compararmos as árvores produzidas pelas classificações ascendentes hierárquicas obtidas a partir das grades de categorização das variáveis descritivas (árvore (1) ) e dos procedimentos matemáticos (árvore (2)) detectamos a existência de um padrão de partição semelhante entre o segundo nível de divisão das duplas na classificação ascendente hierárquica (1) (variáveis descritivas) e a partição das duplas na classificação ascendente hierárquica (2) (procedimentos matemáticos). $\mathrm{O}$ que os dados sugerem, e esta nos parece ser a contribuição central deste estudo, é a evidência empírica de conexão entre dois conjuntos de aspectos referentes ao funcionamento psicológico dos sujeitos analisados: afetividade (aqui operacionalizada em termos da autoestima) e cognição, operacionalizada e especificada em termos do desempenho escolar em matemática (verificada através de ferramenta específica - o teste NAPE).

\section{Conclusões e comentários finais}

O presente estudo identificou a existência de conexões entre aspectos cognitivos e afetivos do desenvolvimento infantil, implicados na questão da aprendizagem, mais especificamente na aprendizagem de conteúdos escolares matemáticos esperados para o primeiro ciclo do Ensino Fundamental. Os dados oriundos desta pesquisa, sistematicamente analisados através de análise categorial de tipo multidimensional, sugerem a existência de uma conexão entre a autoestima e o desempenho escolar em matemática. Tal conexão é claramente percebida na semelhança entre as partições realizadas pela análise 
multidimensional, a partir dos procedimentos e características descritivas das duplas, obtendo-se partição semelhante para ambos os conjuntos de dados (aqueles referentes à autoestima e aqueles referentes ao desempenho em matemática).

Não obstante tal evidência empírica, é preciso prudência na interpretação da conexão aludida, em relação aos dados ora disponíveis: a conexão identificada não nos autoriza a afirmar que haja uma relação de causalidade em uma determinada direção. Não se dispõe, aqui, de dados que permitam afirmar se: (a) a autoestima é determinante e se constitui num preditor do desempenho escolar, ou se (b) o desempenho escolar, por sua vez, desponta como um critério determinante do nível de autoestima apresentado pela criança, ou ainda se (c) haveria relação de causação mútua. O que podemos afirmar, no momento, é a existência de uma conexão empírica entre descritores de natureza afetiva (autoestima) e descritores de natureza cognitiva (desempenho escolar em matemática). Cabe, aliás, comentar que a busca de relações causais unidirecionais simples, como as acima aludidas, não faria justiça ao esforço teórico de busca de abordagem integrada (não dicotômica) para os aspectos afetivos e cognitivos do processo de significação humano. Seria mais produtivo, propomos aqui finalmente, aprofundar as observações aqui obtidas em termos da descrição de contextos de cogênese do funcionamento cognitivo.

\section{REFERÊNCIAS}

ARAÚJO, C. R. et al. Affective aspects on mathematics conceptualization: from dichotomies to an integrated approach. In: INTERNATIONAL CONFERENCE FOR THE PSYCHOLOGY OF MATHEMATICS EDUCATION, 27., 2003, Honolulu, USA. Proceedings... Honolulu, 2003. v. 2, p. 269-276.

ASSOCIATION POUR LE DÉVELOPPMENT DE L'ANALYSE DES DONNÉES - ADDAD. Programas ANCORR ${ }^{\odot}$ (Análise Fatorial de Correspondências), ANCOMP $^{\circ}$ (Análise em Componentes Principais) e CAH ${ }^{\odot}$ (Classification Ascendente Hiérarchique). Paris, 1989.

DA ROCHA FALCÃO, J. T. Contextualizando a educação matemática: uma análise dos parâmetros curriculares nacionais. In: MARCUSCHI, E.; ARAÚJO LIRA SOARES, E. (Orgs.). Avaliação educacional e currículo: inclusão e pluralidade. Recife: Universidade Federal de Pernambuco, 1997. 
DAMÁSIO, A. R. O erro de Descartes: emoção, razão e cérebro humano. São Paulo: Companhia das Letras, 1996.

DOLLE, J. M. De Freud a Piaget. Lisboa: Moraes, 1979.

Para Além de Freud e Piaget - referenciais para novas perspectivas em psicologia. Petrópolis: Vozes, 1993.

FENELON, J-P. Qu'est-ce que l'analyse des données? Paris: Lefonen, 1981.

FIERRO, A. Personalidade e aprendizagem no contexto escolar. In: COLL, C.; PALACIOS, J.: MARCHESI, A. (Orgs.). Desenvolvimento psicológico e educação. Porto Alegre: Martins Fontes, 1996.

FIGUEIREDO, L. C. M. Matrizes do pensamento psicológico. Petrópolis: Vozes, 1996.

FREUD, S. Obras psicológicas completas de Sigmund Freud. Rio de Janeiro: Imago, 1970; 1977.

HAMMER, E. Aplicações clínicas dos desenhos projetivos. São Paulo: Casa do Psicólogo, 1991.

HANNULA, M.; EVANS, J.; PHILIPPOU, G.; ZAN, R. Affect in Mathematics Education - Exploring Theoretical Frameworks. In: JOHNSEN HOINES, M.; FUGLESTAD, A. B. (Orgs.). Proceedings of the PME 28, Bergen, Bergen University College, v. 1, p. 107-132, 2004.

HAZIN, I. Auto-estima e desempenho em matemática: uma contribuição ao debate acerca das relações entre cognição e afetividade. Recife, 2000. Dissertação (Mestrado em Psicologia) não publicada - Departamento de Psicologia, Pós-Graduação em Psicologia, CFCH/UFPE.

HAZIN, I.; DA ROCHA FALCÃO, J.T. Self-esteem and performance in school mathematics: a contribution to the debate about the relationship between cognition and affect. In: ENCONTRO INTERNACIONAL DO GRUPO PSYCHOLOGY OF MATHEMATICS EDUCATION (PME), 25, 2001, Utrecht (Holanda). Anais... Utrecht, 2001.

LAKOFF, G.; NÚÑEZ, R. E. Where mathematics comes from: how the embodied mind brings mathematics into being. New York: Basic Books, 2000.

MALMIVUORI, M.-L. A Dynamic Viewpiont: Affect in the Functioning of Self-System Processes. In: JOHNSEN HOINES M.; FUGLESTAD, A. B. (Orgs.). Proceedings of the PME 28, Bergen: Bergen University College, v. 1, p. 118, 2004. 
HAZIN, I.; FRADE, C.; FALCÃO, J. T. da R. Autoestima e desempenho escolar...

MEC; SAEB - Sistema Nacional de Avaliação da Educação Básica - Matemática. Disponível em: <http://www.inep.gov.br/download/saeb/2001/relatorioSAEB_ matematica.pdf>. Acesso em: 2001; 2002.

PIAGET, J. Biologia e conhecimento: ensaio sobre as relações entre as regulações orgânicas e os processos cognoscitivos. Petrópolis: Vozes, 1973.

. Epistemologia Genética. São Paulo: Martins Fontes, 1990.

ROAZZI, A. Categorização, formação de conceitos e processos de construção de mundo: procedimentos de classificações múltiplas para o estudo de sistemas conceituais e sua forma de análise através de métodos multidimensionais. Cadernos de psicologia, n. 1, p. 1-27, 1995.

SCHLÖGLMANN, W. Affect and mathematics learning. In: COCKBURN, A. D.; NARDI, E. (Orgs.). Proceedings of the PME 26, Norwich University of East Anglia, v. 4, p. 185-192, 2002.

. Meta-affect and strategies in mathematics learning. Disponível em: <http:// cerme4.crm.es/Papers\%20definitius/2/WolfgangSchloeglmann.pdf $>$. Acesso em: 2007.

SOUZA, M. T. C. C. O desenvolvimento segundo Piaget. In: ARANTES, V. A. Afetividade na escola: alternativas teóricas e práticas. São Paulo: Summus, 2003.

VYGOTSKY, L. S. A construção do pensamento e da linguagem. São Paulo: Martins Fontes, 2001.

. Obras escogidas. v. 2. Madrid: Visor, 1993.

WERTHEIMER, M. Productive thinking. New York: Harper and Row, 1959.

ZAN, R.; BROWN, L.; EVANS, J.; HANNULA, M. S. (Orgs.). Special Issue on Affect in Mathematics Education. Educational Studies in Mathematic,. 2006.

Texto recebido em 19 de novembro de 2009.

Texto aprovado em 12 de janeiro de 2010. 